\title{
LETTERS
}

\section{Predictive value of latent tuberculosis tests in Indian healthcare workers: a cohort study}

\section{To the Editors:}

Healthcare workers (HCWs) in high tuberculosis (TB)-burden countries are known to have a substantially higher risk of latent TB infection (LTBI) and TB disease [1], and this has been demonstrated repeatedly in India [2,3]. Several studies have evaluated interferon- $\gamma$ release assays (IGRAs) in HCWs [4], but no study has reported the predictive value of IGRAs in this population of HCWs at risk for TB exposure.

Between January and June 2004, we had performed a crosssectional comparison of the tuberculin skin test (TST)
(1 tuberculin unit of purified protein derivation RT23) and QuantiFERON ${ }_{\circledR}$-TB Gold In-Tube (QFT; Cellestis Ltd, Melbourne, Australia) in a cohort of 726 HCWs (719 had valid test results for both tests) at the Mahatma Gandhi Institute of Medical Sciences (Sevagram, India), with young trainees making up half the cohort [5]. A total of 360 (50\%) HCWs were found to be positive using either the TST or QFT assay at baseline, and 226 (31\%) were found to be positive using both tests. The prevalence estimates of TST and QFT positivity were comparable, with high concordance between test results [5]. Although isoniazid preventive therapy (IPT) was offered to

TABLE 1 Incidence of active tuberculosis (TB) disease according to baseline variables ${ }^{\#}$

\begin{tabular}{|c|c|c|c|c|}
\hline $\begin{array}{l}\text { Baseline } \\
\text { exposure variables }\end{array}$ & TB disease events $n$ & $\begin{array}{l}\text { Follow-up } \\
\text { person-yrs }\end{array}$ & $\begin{array}{l}\text { Incidence per } 1000 \text { person-yrs } \\
\qquad(95 \% \mathrm{Cl})\end{array}$ & IRR $^{\star}(95 \% \mathrm{CI})$ \\
\hline $18-20$ & 4 & 1246.75 & $3.21(1.20-8.55)$ & 1.00 \\
\hline $21-30$ & 9 & 1731.25 & $5.20(2.70-9.99)$ & $1.62(0.45-7.2)$ \\
\hline $31-40$ & 0 & 432.00 & & \\
\hline Males & 4 & 1559.67 & $2.56(0.96-6.83)$ & 1.00 \\
\hline Females & 10 & 2432.42 & $4.11(2.21-7.64)$ & $1.60(0.46-7.00)$ \\
\hline \multicolumn{5}{|l|}{ Job category } \\
\hline Trainees & 10 & 2333.75 & $4.28(2.31-7.96)$ & 1.00 \\
\hline Nurses & 4 & 856.33 & $4.67(1.75-12.45)$ & $1.09(0.24-3.77)$ \\
\hline Physicians & 0 & 72.00 & & \\
\hline $6-10$ & 2 & 666.50 & $3.00(0.75-12.00)$ & $0.64(0.06-2.94)$ \\
\hline$>10$ & 1 & 972.08 & $1.03(0.14-7.30)$ & $0.22(0.01-1.51)$ \\
\hline \multicolumn{5}{|l|}{ TST result } \\
\hline Negative $^{\S}$ & 8 & 2337.25 & $3.42(1.71-6.84)$ & 1.00 \\
\hline Positive ${ }^{f}$ & 6 & 1654.83 & $3.63(1.63-8.07)$ & $1.05(0.30-3.48)$ \\
\hline \multicolumn{5}{|l|}{ QFT result } \\
\hline Negative ${ }^{\# \#}$ & 8 & 2365.75 & $3.38(1.69-6.76)$ & 1.00 \\
\hline Positive" & 6 & 1626.33 & $3.69(1.66-8.21)$ & $1.09(0.31-3.58)$ \\
\hline \multicolumn{5}{|l|}{ TST and QFT results } \\
\hline Concordant negative & 7 & 1991.67 & $3.51(1.68-7.37)$ & 1.00 \\
\hline Discordant & 2 & 719.67 & $2.78(0.70-11.11)$ & $0.79(0.08-4.15)$ \\
\hline Concordant positive & 5 & 1280.75 & $3.90(1.62-9.38)$ & $1.11(0.27-4.06)$ \\
\hline
\end{tabular}

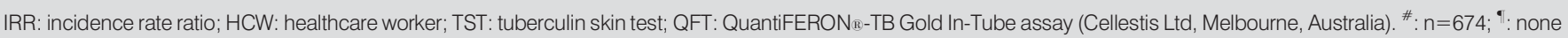

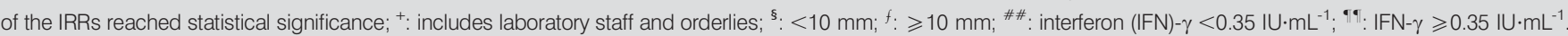




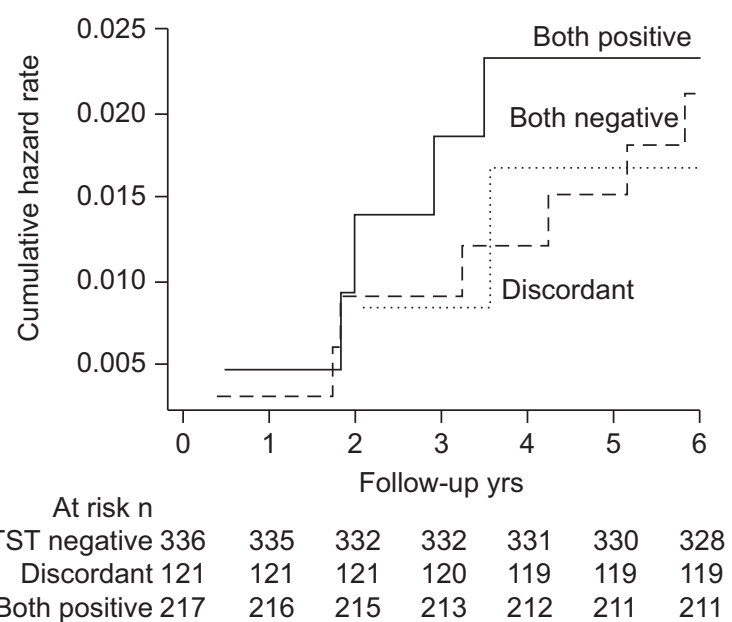

FIGURE 1. Cumulative hazard function for occurrence of active tuberculosis across tuberculin skin test (TST) and QuantiFERON ${ }_{\mathbb{B}}$-TB Gold In-Tube (QFT; Cellestis Ltd, Melbourne, Australia) result categories $(n=674)$. Incidence rate ratio among both TST- and QFT-positive, as compared with those with both negatives test results, at end of 2, 4 and 6 yrs is 1.54 (95\% Cl 0.20-11.55), 1.93 (95\% Cl 0.419.77 ) and 1.11 (95\% $\mathrm{Cl} 0.27-4.06)$, respectively. Cumulative hazard rate in these two subgroups is statistically similar (log-rank test $p=0.84$ ).

HCWs with positive baseline TST or QFT results, only a small proportion had completed INH therapy.

In 2010, 6 yrs after the baseline survey, we followed-up the HCWs, using face-to-face interviews, and telephone or e-mail contact. All participants who had developed active TB disease were asked about the date of their diagnosis, nature of their TB disease and evidence of diagnosis.

We estimated the incidence rate of active TB disease (per 1,000 person-yrs) for the entire cohort, across job categories and subgroups with positive or negative TST or IGRA results, using standard definitions for a positive TST $(\geqslant 10 \mathrm{~mm})$ and QFT (interferon (IFN)- $\gamma \geqslant 0.35 \mathrm{IU} \cdot \mathrm{mL}^{-1}$ ) test result. We estimated the incidence rate ratio (IRR) as the ratio of $\mathrm{TB}$ incidence in the exposed versus unexposed categories.

In 2010, we successfully contacted 674 (94\%) out of 719 participants to ascertain the incidence of active TB disease. Of the 674 HCWs followed for 3,992.08 person-yrs, 14 had developed active TB disease (incidence rate 3.50, 95\% CI 2.075.92 per 1,000 person-yrs (table 1), twice that of the Indian general population incidence of 1.70 per 1,000 person-yrs [6]). Four $(28.6 \%)$ HCWs had developed pulmonary TB, while the remaining 10 had extra-pulmonary TB (four pleural, four lymph node, one abdominal and one bone/joint TB). A total of six $(43 \%)$ out of 14 HCWs had evidence of smear- and/or culturepositive TB, and almost all of them were $<30$ yrs of age. The incidence of TB was high in young trainees, nurses and those who had worked for $<5$ yrs as a HCW at the time of baseline survey, although none of the IRRs reached significance (table 1).

Incidence rates of TB disease in the TST and QFT positive and negative subgroups were similar, and IRRs were close to 1.0 (null) (1.05 (95\% CI 0.30-3.48) and 1.09 (95\% CI 0.31-3.58) for TST and QFT, respectively). HCWs with concordant positive test results were not at a statistically significant higher risk at any intermediate time interval during follow-up (fig. 1). HCWs who had developed active TB had similar median TST induration sizes or IFN- $\gamma$ levels at baseline as those who did not progress to disease (data not shown).

In a high-burden setting, our data showed that Indian HCWs were twice as likely to develop active TB as the general population, and young trainees were particularly at risk and needed to be targeted for screening and preventive therapy. Our study also showed that a single positive QFT or TST test result at baseline, when used in isolation, did not significantly predict future risk of active TB during 6 yrs of follow-up. The predictive ability of the QFT test was similar to that of the TST, and with both tests, a large proportion of those with baseline positive results did not progress to TB disease. This is consistent with similar cohort studies from other high TB-burden countries, including South Africa [7], Gambia [8], Turkey [9], Senegal [10] and Colombia [11], which showed the comparable predictive ability of IGRA and TST. Thus, all existing LTBI tests (TST and IGRAs) may have only modest predictive value and may not identify those who are at highest risk of progression to TB disease in endemic countries.

In contrast, IGRAs appear to be more predictive in low TBincidence settings, as shown in studies from Austria [12] and Germany [13]. In general, IGRAs appear to perform better in low versus high TB-incidence settings. It is likely that repeated exposures in high TB-incidence settings attenuate the predictive ability of a single cross-sectional TST or IGRA result. In addition, without performing serial testing with TST or IGRAs, it is impossible to determine the extent of exposure or whether the infection was recent (i.e. conversion), one of the most important factors that predict risk of progression [14].

There is growing recognition that LTBI is a spectrum, and accumulating evidence suggests that none of the existing LTBI tests can resolve this spectrum [15, 16], particularly with onetime testing [14]. It is likely that a proportion of individuals who become infected with Mycobacterium tuberculosis eliminate all live TB bacteria and are, thus, no longer "infected", while they still have an adaptive immune response to the TST or IGRA, perhaps to a lesser extent with IGRAs [16]. Thus, current LTBI tests may not be able to separate out the underlying phenotypes and identify the subset that is at highest risk of future disease, as confirmed by a new meta-analysis [17]. Therefore, the search for more predictive biomarkers or combinations of biomarkers and risk factors must continue.

\section{R. Joshi*, U. Narang*, A. Zwerling", D. Jain*, V. Jain*, S. Kalantri* and M. Pai ${ }^{*}$}

*Dept of Medicine, Mahatma Gandhi Institute of Medical Sciences, Sevagram, India. "Dept of Epidemiology and Biostatistics, McGill University, Montreal, QC, Canada.

Correspondence: M. Pai, Dept of Epidemiology and Biostatistics, McGill University, 1020 Pine Ave West, Montreal, QC H3A 1A2, Canada. E-mail: madhukar.pai@mcgill.ca

Support Statement: This study was supported by a grant from the Canadian Institutes of Health Research (CIHR; grant MOP-81362). This agency had no role in the design, execution or publication of this study. M. Pai is supported by a New 
Investigator Award from CIHR and A. Zwerling is supported by a CIHR doctoral fellowship award.

Statement of Interest: None declared.

\section{REFERENCES}

1 Joshi R, Reingold AL, Menzies D, et al. Tuberculosis among healthcare workers in low- and middle-income countries: a systematic review. PLoS Med 2006; 3: e494.

2 Pai M, Joshi R, Dogra S, et al. Serial testing of health care workers for tuberculosis using interferon- $\gamma$ assay. Am J Respir Crit Care Med 2006; 174: 349-355.

3 Christopher DJ, Daley P, Armstrong L, et al. Tuberculosis infection among young nursing trainees in South India. PLoS One 2010; 5: e10408.

4 Zwerling A, van den Hof S, Scholten J, et al. Interferon- $\gamma$ release assays for tuberculosis screening of healthcare workers: a systematic review. Thorax 2011; [Epub ahead of print DOI: 10.1136/thx.2010.143180].

5 Pai M, Gokhale K, Joshi R, et al. Mycobacterium tuberculosis infection in health care workers in rural India: comparison of a whole-blood interferon gamma assay with tuberculin skin testing. JAMA 2005; 293: 2746-2755.

6 World Health Organization. TB Data: Data Collected from TB Control Programmes and Estimates Generated by WHO. www. who.int/tb/country/data/download/en/index.html Date last accessed: October 30, 2010. Date last updated: 2010.

7 Mahomed H, Hawkridge T, Verver S, et al. The tuberculin skin test versus QuantiFERON TB Gold $₫$ in predicting tuberculosis disease in an adolescent cohort study in South Africa. PLoS One 2011; 6: e17984.

8 Hill PC, Jackson-Sillah DJ, Fox A, et al. Incidence of tuberculosis and the predictive value of ELISPOT and Mantoux tests in Gambian case contacts. PLoS One 2008; 3: e1379.
9 Bakir M, Millington KA, Soysal A, et al. Prognostic value of a Tcell-based, interferon- $\gamma$ biomarker in children with tuberculosis contact. Ann Intern Med 2008; 149: 777-787.

10 Lienhardt C, Fielding K, Hane AA, et al. Evaluation of the prognostic value of IFN- $\gamma$ release assay and tuberculin skin test in household contacts of infectious tuberculosis cases in Senegal. PLoS One 2010; 5: e10508.

11 del Corral $\mathrm{H}$, Paris SC, Marin ND, et al. IFN $\gamma$ response to Mycobacterium tuberculosis, risk of infection and disease in household contacts of tuberculosis patients in Colombia. PLoS One 2009; 4: e8257.

12 Aichelburg MC, Rieger A, Breitenecker F, et al. Detection and prediction of active tuberculosis disease by a whole-blood interferon- $\gamma$ release assay in HIV-1-infected individuals. Clin Infect Dis 2009; 48: 954-962.

13 Diel R, Loddenkemper R, Niemann S, et al. Negative and positive predictive value of a whole-blood interferon- $\gamma$ release assay for developing active tuberculosis: an update. Am J Respir Crit Care Med 2010; 183: 88-95.

14 Pai M. Spectrum of latent tuberculosis - existing tests cannot resolve the underlying phenotypes. Nat Rev Microbiol 2010; 8: 242.

15 Barry CE, Boshoff HI, Dartois V, et al. The spectrum of latent tuberculosis: rethinking the biology and intervention strategies. Nat Rev Microbiol 2009; 7: 845-855.

16 Mack U, Migliori GB, Sester M, et al. LTBI: latent tuberculosis infection or lasting immune responses to $M$. tuberculosis? A TBNET consensus statement. Eur Respir J 2009; 33: 956-973.

17 Rangaka MX, Wilkinson KA, Glynn JR, et al. Predictive value of interferon $-\gamma$ release assays for incident active tuberculosis: a systematic review and meta-analysis. Lancet Infect Dis 2011; [Epub ahead of print DOI: 10.1016/S1473-3099(11)70210-9].

DOl: $10.1183 / 09031936.00014611$

\section{Microsatellite alterations at $3 p$ and $19 q$ in EBC DNA of smokers: are they reversible after smoking cessation?}

\section{To the Editors:}

Carcinogens in cigarette smoke may leave fingerprints in the bronchial tissue in the form of specific mutations that initiate cancer development [1]. Numerous genetic alterations have been recognised as critical effects of cigarette smoke and studied in the airways of current and former smokers. Microsatellite alterations (MAs) at 3p and 19q are among the most studied, being considered useful markers of genetic susceptibility and genome destabilisation in susceptible smokers [2, 3]. Instability and/or loss of heterozygosity at $3 p$ and $19 q$ have largely been reported in the lung tissue, sputum and blood of smokers and lung tumour patients $[2,4]$. These mutations, which are considered an early effect of cigarette smoke, are dose-dependent and related to the number of cigarettes smoked in a lifetime [3]. In addition, short-term exposure to cigarette smoke seems to cause MAs at $3 p$ that are not necessarily a consequence of the development of a neoplastic mass [5].

Our group recently reported MAs at these loci in the exhaled breath condensate $(\mathrm{EBC})$ of smokers and patients affected by nonsmall cell lung cancer, demonstrating that EBC could be a surrogate for tissue in assessing tobacco-induced molecular damage in the lungs [2,3].

Interestingly, although smoking cessation may reverse the clonal expansion of abnormal cells, the persistence of some genetic alterations in former smokers indicates that a high rate of clonal genetic damage persists even after smoking cessation [6].

Several genetic susceptibility markers have been studied after smoking cessation and classified as rapidly reversible, slowly reversible or irreversible [7]. Indeed, to know whether a gene alteration is reversible could turn out to be important, as rapidly reversible genes seem to have different biological functions than slowly reversible or irreversible genes [7].

The aim of this study was to test the reversibility of MAs at $3 p$ and $19 q$ by analysing these markers for the first time in the EBC and whole blood (WB) of smokers at baseline (T0) and then at 12 months after smoking cessation (T1). The correlations between MAs with sex, pack-yrs, exhaled carbon monoxide and Fagerström nicotine dependence score were also investigated. 